\title{
VIEWPOINT
}

\section{Predicting Coma Trajectories: The Impact of Bias and Noise on Shared Decisions}

\author{
Eelco F. M. Wijdicks ${ }^{1,2,3^{*}}$ and David Y. Hwang ${ }^{1,2}$ \\ (C) 2021 Springer Science+Business Media, LLC, part of Springer Nature and Neurocritical Care Society
}

\begin{abstract}
Coma trajectories are characterized by quick awakening or protracted awakening. Outcome is bookended by restored functionality or permanent cognitively and physically debilitated states. Given the stakes, prognostication cannot be easily questioned as a judgment call, and a scientific underpinning is elemental. Conventional wisdom in determining coma-outcome trajectories posits that (1) predictive models are better than personal experiences, (2) self-fulfilling prophesy is unchecked and driven by nihilism, with little regard for prior probability outcomes, and (3) recovery is impacted by patients' prior wishes and preexisting medical conditions - but also by what families are told about the patient's state and anticipated clinical course. Moreover, a predicted good outcome can be offset by a major subsequent complication, or a predicted poor outcome can be offset by aggressive care. This article examines some of these concepts, including how we decide on aggressiveness of care, how we judge quality of life, and the impact on outcome. Most patients who awaken quickly do well and can resume their pretrauma injury lives. In worse off, slowto-awaken patients, outcomes are a mixed bag of limited innate resilience, depleted cognitive and physical reserves, and adjusted quality of life. Bias and noise are factors not easily measured in outcome prediction, but their influence on recovery trajectories raises some troubling issues.
\end{abstract}

Keywords: Coma, Outcome, Bias, Decisions, Conferences, Families

\section{Introduction}

Predicting recovery trajectories in acute brain injuryinduced coma presents a major clinical problem. We rely on repeated neurologic examinations (before and after a major intervention), our experiences, published literature, and emerging new technology $[1,2]$. We recognize the misconception of assuming these trajectories are predictably fixed and understand that there are shortcomings in our assumptions of outcome [3]. Prognostication in coma recovery differs greatly from other areas in medicine (such as oncology, with its numerous KaplanMeier survival probability curves and general critical care with different population sets [4]). Less-recognized confounders that influence decisions can be numerous [5].

\footnotetext{
*Correspondence: wijde@mayo.edu

${ }^{3}$ Division of Neurocritical Care and Hospital Neurology, Mayo Clinic, 200

First Street SW, Rochester, MN 55905, USA

Full list of author information is available at the end of the article
}

Several factors, in some way of form, can seriously bend the recovery trajectory curve, and we like to point out, based on our experiences with family interactions, that they should be recognized (and, if feasible, measured) in studies on coma recovery.

\section{General Considerations with Prediction of Coma Trajectories}

First, improvements in overall outcomes in the field of neurocritical care may outpace those predicted by published outcome scales. Looking back over the past 50 years (when outcome scales in brain injury first started to appear), outcomes have improved as a result of small incremental benefits from multiple factors, e.g., the intensity of care or the sum of the number of interventions [6, 7]. Bureaucracy-driven healthcare that systematically tracks infections and implements other hospital safety measures may have contributed. 
Therefore, available literature on outcome may quickly become or have already become obsolete.

Second, variability in aggressiveness of care for patients with devastating brain injury leads to variability in mortality and opportunities for improvement for some patients. For example, mortality associated with withdrawal of life-sustaining therapy in severe traumatic brain injury varies in centers [8-10], and eventual recovery from disorders of consciousness is expected in patients selected for rehabilitation centers [11]. Moreover, indications for neurosurgical interventions differ across centers. Earlier studies on preferences and equipoise have shown that neurosurgeons were more certain about surgical choices for older patients, patients with a higher Glasgow Coma Scale, and patients in whom the hematoma was located on in the nondominant hemisphere, in the basal ganglia, or in the thalamus. Patients for whom the neurosurgeon was certain about treatment were more likely to have the hematoma removed if they were younger [12]. In another study, postoperative patient management strategies, including the use of intracranial pressure monitoring, cerebrospinal fluid drainage, and mechanical ventilation, varied among centers, but their overall rate of surgical intervention was low at $5 \%$ [13].

Third, in recovery of acute brain injury, nothing cannot be predicted with certainty, and we should harbor no illusions. No accepted easy tools (or even less easy tools, such as artificial intelligence) reliably estimate recovery of consciousness in comatose patients. Furthermore, the exact trajectory of coma recovery is often K-shaped (Fig. 1). Patients' paths diverge like the arms of the letter "K"; some follow a relentlessly downward trajectory, whereas others go up and improve day by day. Although excellent pre-illness functionality creates the best opportunities and poor pre-illness states may set a patient back, uncertainty regarding an individual patient's exact outcome is most often par for the course. In our experience, most patients in a poor functional state may recover but will permanently have lost more functionality.

Fourth, only recently has the term "self-fulfilling prophecy" played an important role in questioning the accuracy of prognostication. Sociologist Robert Merton coined the expression in 1948, defining it as "a false definition of the situation evoking a new behavior which makes the original false conception come true" [14]. In assessment of outcome studies on the natural history of an acute brain injury, a bias toward a poor outcome could have led to unjustifiable discontinuation of supportive care, and thus the underlying theory of self-fulfilling prophecy is that prediction affects outcome. In other words, an unjustifiably negative prognosis may lead to withdrawal of support and, therefore, a poor outcome. Possibly, these

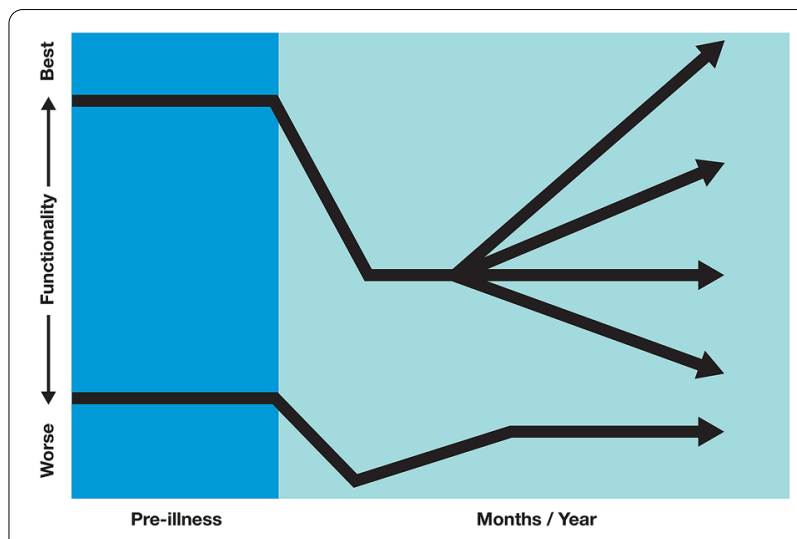

Fig. 1 Outcome trajectories. Most published outcomes do not go beyond a year. We expect a number of good recoveries, but patients with a poor functional baseline rarely return to their baseline condition

"Cassandras" contribute to mortality rates of patients with stroke, traumatic brain injury, and anoxic-ischemic encephalopathy $[15,16]$. Proponents of this hypothesis feel that an approach of going the extra mile in terms of resuscitative measures and aggressive care usually leads to improvement of the patient. A patient with a poor grade subarachnoid hemorrhage can progress to a good grade with a ventriculostomy, and a patient rapidly deteriorating from an expanding cerebral hematoma (particularly if supratentorial or infratentorial lobar) may improve very quickly after clot evacuation.

However, regarding avoiding self-fulfilling prophecies, there are important nuances; we must be careful not to suggest it is rampant in neurocritical care. Evidence suggests that neurointensivists, as a profession, generally err on the side of optimism regarding prognostic errors [17]. The other side of self-fulfilling prophecy is that hope springs eternal, leading to false hope. There are times when a pessimistic prognosis is justified. A few examples suffice: a resuscitated, comatose, cardiac arrest patient with diffuse cortical injury on Magnetic resonance imaging, increasing serum neuron-specific enolase values, nonreactive Electroencephalogram (EEG) with poor background, and no cortical responses on somatosensory evoked potentials (SSEP). None of these patients are likely to have a good outcome. Similarly, a devastated trauma patient with large bilateral cerebral contusions, refractory increased intracranial pressure, and loss of most brainstem reflexes cannot have a good outcome.

Doing everything medically possible may lead to an irreversible, devastating disability rather than a good quality of life. Therefore, both overly optimistic and pessimistic attitudes (overall or for a specific case) may warp decision-making considerations. No neurointensivist 
wants to deprive the patient or family of a possible good outcome or deprive them of hope. Focusing on the possibility of the self-fulfilling prophecy may lead clinicians to feel responsible for the death of their patients and can create a fear of causing, or even hastening, death. Avoidance of self-fulfilling prophecy may lead to continuation of treatment and full resuscitative measures in situations that are not in a patient's best interest. This may be of particular concern for the older population, for whom a coma-induced disability may increase the burden of age-induced disability. Doing everything medically possible for many comatose patients with brain injury may result in an irreversible, devastating disability rather than an anticipated good quality of life. Yet, indiscriminately, physicians may be far more comfortable with treating early and withdrawing later.

\section{Quality of life}

"Quality of life" is subjective and multifactorial (Fig. 2), and the term stubbornly resists explanations that reduce it to a couple of criteria. There are as many definitions of quality of life as there are people trying to define it. We assume that many clinicians often do not hesitate to use "poor quality of life" in their discussions with families to describe what they personally anticipate to be the experience of an extremely devastated patient with brain injury with no or slow recovery. These are in general absent sense of purpose, a need for constant help, loneliness, sadness, worthlessness, immobility, and no recollection of recent events. However, there is a serious concern about overreach if we present poor quality of life as a life "not worth living" with certainty, especially when significant prognostic uncertainty exists [18].

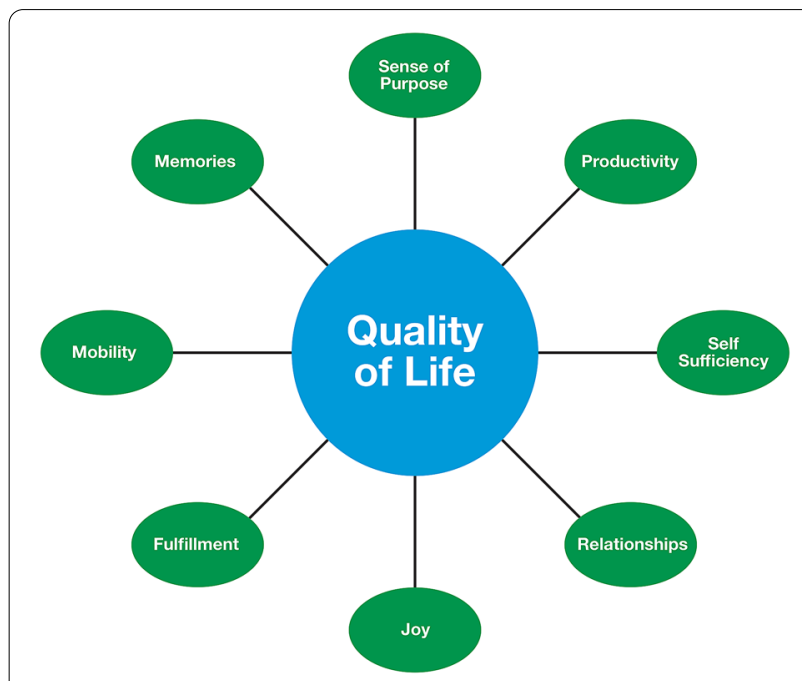

Fig. 2 Some domains that determine quality of life
The task of affective forecasting, predicting not just how a person will be in the future but how they will feel about their situation, is challenging [19]. How much can a person adjust to disability or find new meaning in a drastically altered life? Will the loss of many pleasurable and meaningful activities cause a joyless existence? For some, the inability to focus on a complex task may already be devastating. Traditionally, clinicians considered the absence of major medical signs and symptoms as a major index of quality of life, but we all know there is much more. In devastated patients, there is no mind without mindfulness.

Families share in the decision-making process, which includes the involvement in most elements of care, but also share their views on what can be an acceptable quality of life for their loved one. They can do it because they know the person and have a good sense of what they would have wanted. Clinicians have come to understand that they cannot adjudicate normality and abnormality when it pertains to how a patient might feel in a future state and should avoid judging quality of life. To conclude that the person's quality of life is going to be acceptable or unacceptable is now, fortunately, a consensus opinion without any person, including the clinician, taking a dominant role. Nonetheless, a disability paradox may exist, which posits that young, healthy persons rank the quality of life of a disabled person much lower on the scale than the recently rehabilitated, disabled person ranks their own quality of life. This phenomenon has been shown to be present across several types of acute brain injuries and has practical and ethical consequences.

\section{Families Concerns and Expectations}

For families, various concerns surround the question foremost on their minds: will their loved one wake up? This includes concerns of whether prognostication will be accurate. Of note, the format of how clinicians present prognostic data significantly influences how surrogate decision-makers of patients in the intensive care unit (ICU) interpret risk. Quantitative prognostic statements presented in frequency (numerical) format (e.g., 1 of 50) are perceived as higher risk compared with the equivalent percentages (e.g., 2\%) [20]. Visual thinking has a profound impact on the way we approach prognostication and may start with showing neuroimaging, which will be dramatic for the uninitiated. Graphic representations may make numbers more comprehensive for families but have their own problems, as outlined in Fig. 3.

Regardless of how clinicians present their opinions, family members of patients in the ICU cite some factors contributing to their own prognostic estimates for their loved ones, such as (1) knowledge of the patient's strength of character and will to live, (2) the patient's 

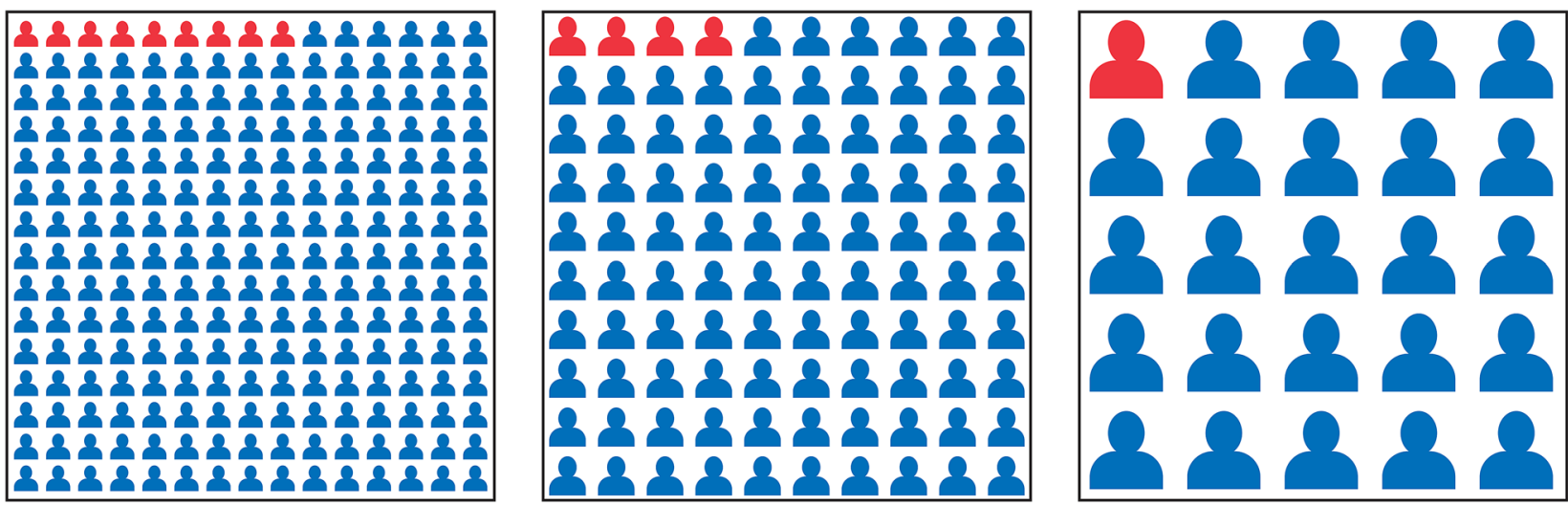

Fig. 3 Examples of graphs that can be used to show poor outcome (in red). We use $4 \%$ as an example. Note that many would likely believe the right graph (1 in 25) suggests a higher risk of poor outcome than the other graphs (9 in 225 and 4 in 100), when in fact all are exactly the same (Color figure online)

history of illness and survival, (3) the surrogate's observation of the patient's appearance and status, (4) the surrogate's belief that their own actions and presence at the bedside might influence prognosis, and (5) the surrogate's optimism, intuition, and faith [21]. When presented a hypothetical scenario of making an ICU goals-of-care decision for a loved one with a severe acute brain injury and poor prognosis, Americans who were more inclined toward tracheostomy and feeding-tube placement were young, men, evangelical Christian or Catholic, and in a lower income bracket [22]. Aside from accurate prognostication, a significant proportion of all potential surrogate decision-makers in the same US survey study reported family consensus as a major concern amid decision-making, as well as how to pay for long-term care [23].

What more do families expect of decision-making support in neuroscience ICUs? As it turns out, there is significant room for improvement [24]. Using support groups for family members of patients admitted to an ICU may complement other types of support offered to the family. Family support coordinators may improve communication between ICU clinicians and families, thus increasing family satisfaction [25]. Open or flexible visiting hours for families of patients in the ICU improve family satisfaction, and family participation in ward rounds increases frequency of communication with ICU clinicians. Families appreciate onsite guest rooms within the hospital to spend the night or gather with other family members [26].

\section{Conclusions}

Recovery from coma usually occurs in stages: awakening, awareness, communication, and, later, return of an inner urge to become more dynamic, resulting in focus and motivation to reach new physical and mental objectives and goals. None of these stages is completely predictable, let alone clearly outlined, and they are etiology dependent. If there is no primary brain injury (such as in an overdose, diabetic coma, or a major respiratory illness), even after days on the ventilator, recovery from coma is understandably rapid. Other patients' recoveries are largely determined by the extent of their brain injury and associated consequences, such as ventilation or respiration difficulties.

Ultimately, we inform family members and make the best shared decisions we can. Each patient will eventually fall into one of the categories in Fig. 4; the figure delineates reasonable approaches in the care of a seriously afflicted person, and all must be assumed goal-concordant or preference-sensitive. Our general principle is to postpone final goals-of-care decisions until after some period of maximal care unless it is a devastating injury from the very start. First, in most patients we just do what we need to do and do what we should do (Fig. 4a), Second, in some patients, we apply short-term all systems go aggressive care ("full court press"), knowing that if it is not successful, we will proceed with gradual de-escalation; i.e., if there is no change in the neurologic condition and if family agrees with less-aggressive measures (Fig. 4b). In those situations, we pursue the practice of critical care and palliative care in parallel to the extent that it is possible. The more we let go of aggressive critical care, the more we need to increase appropriate comfort measures. Third, we undertake a more abrupt transition (Fig. 4c), often after deciding to give it (i.e., any feasible medical or surgical option) one more try and then quickly abandon critical care if the "hail Mary" attempt is unsuccessful. Fourth, we pursue aggressive care with all resuscitative measures followed by continued critical care, but when an unexpected major complication occurs, we 


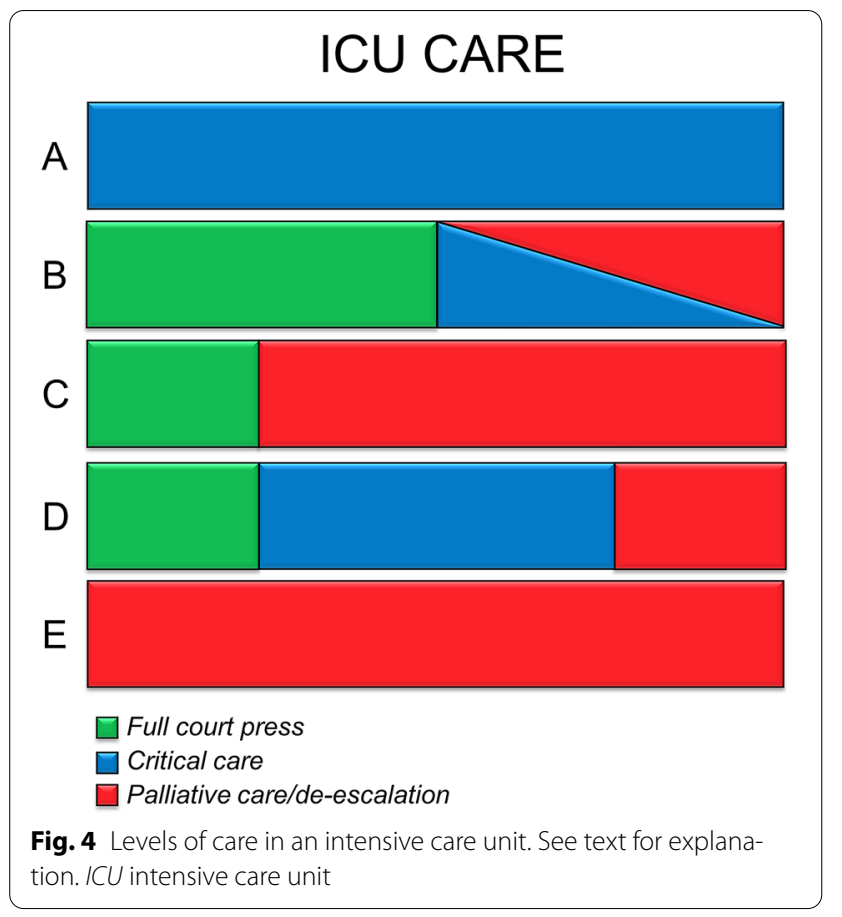

decide to provide palliation only [27] (Fig. 4d). Finally, palliative care may be initiated immediately, and patients are not transferred to ICUs (Fig. 4e). Each of these approaches influences coma recovery trajectories. The "elephant in the room" is when incongruency in clinical assessment appears (family sees progress this way and we see it another way). There are many branching points and decisions in a patient trajectory that could cumulatively lead to a wide divergence [28]. Families and the health care team may draw opposite conclusions from the same evidence with persistent disagreements.

The practice of medicine, particularly neurology, is also based on skepticism and self-inspection. When we examine and care for a patient, clinicians are naturally full of doubt about what we find. We, the neurodissenters, question continuously whether we are responding correctly to what we see on examination, and we always ask ourselves, "Should we do it differently?" Clinicians are not all-knowing; judgments may seem like a shot in the dark, and we carry the stress of prediction. We are humbled if we are proven wrong in both ways (i.e., expecting poor outcome and then the patient recovers, or expecting good outcome in a patient who fares poorly). Some clinicians may agonize over their own or others' judgments, even more so if there are glaring mistakes [29]. But predicting outcome is not done out of arrogance or hubris; without our best attempts at outcome prediction, we may seriously harm the patient by continuing care against all odds or limiting care prematurely.
We should warn against healthcare workers who admit that the data are extremely limited but continue to insist that they support their prediction. We should warn against knee-jerk oversimplification and realize that we are all human beings and dependent, in part, on our current mood. Without assigning a number to it, we should always ask ourselves, "What are the odds?" Moreover, we should be reluctant doomsayers, although we must pay attention to inauspicious information in some truly bad situations. Again, most patients who awaken quickly do well, whereas patients who are severely injured or slowto-awaken present significant ambiguity. Other factors, such as intuition, prior negative experiences, mood, tiredness, and secondary interest (avoidance of mortality counting as poor performance), influence clinician outcome prediction but are not easily measured. Prognostication by artificial intelligence could, in theory, be better than clinicians' subjective judgment, but we would need a sheer magnitude of data-with details and more details. We may have to conclude that prediction of a coma trajectory is ultimately defined by a large number of variables that cannot be known to a physician or computer.

\section{Author details \\ ${ }^{1}$ Neuroscience Intensive Care Units, Saint Marys Hospital, Mayo Clinic Campus, Rochester, MN, USA. ${ }^{2}$ Yale New Haven Hospital, New Haven, CT, USA. ${ }^{3}$ Divi- sion of Neurocritical Care and Hospital Neurology, Mayo Clinic, 200 First Street SW, Rochester, MN 55905, USA. \\ Author contributions \\ Drs. EFMW and DYH contributed equally (conceptualized, analyzed and acquired data, and drafted), and approved the final manuscript.}

\section{Source of support}

No extramural funding supported the preparation of this article.

\section{Declarations}

\section{Conflicts of interest}

Neither author reports relevant conflicts.

\section{Ethical approval/informed consent}

This is a Viewpoint article and discusses no specific patients or cohorts; thus, institutional review board approval was not necessary.

\section{Publisher's Note}

Springer Nature remains neutral with regard to jurisdictional claims in published maps and institutional affiliations.

Received: 21 June 2021 Accepted: 28 July 2021

Published online: 23 August 2021

\section{References}

1. Edlow BL, Chatelle C, Spencer CA, et al. Early detection of consciousness in patients with acute severe traumatic brain injury. Brain. 2017;140(9):2399-414.

2. Edlow BL, Claassen J, Schiff ND, et al. Recovery from disorders of consciousness: mechanisms, prognosis and emerging therapies. Nat Rev Neurol. 2021;17(3):135-56. 
3. Wartenberg KE, Hwang DY, Haeusler KG, et al. Gap analysis regarding prognostication in neurocritical care: a joint statement from the German neurocritical care society and the neurocritical care society. Neurocrit Care. 2019;31(2):231-44

4. Truog RD, Cist AF, Brackett SE, et al. Recommendations for end-of-life care in the intensive care unit: the Ethics Committee of the society of critical care medicine. Crit Care Med. 2001;29(12):2332-48.

5. Griesdale D, Jones PM. In asking the right questions, be cautious of confounding by indication. Can J Anaesth. 2018;65(9):979-84.

6. Bershad EM, Feen ES, Hernandez $\mathrm{OH}$, et al. Impact of a specialized neurointensive care team on outcomes of critically ill acute ischemic stroke patients. Neurocrit Care. 2008;9(3):287-92.

7. McCredie VA, Alali AS, Scales DC, et al. Impact of ICU structure and processes of care on outcomes after severe traumatic brain injury: a multicenter cohort study. Crit Care Med. 2018;46(7):1139-49.

8. Cote N, Turgeon AF, Lauzier F, et al. Factors associated with the withdrawal of life-sustaining therapies in patients with severe traumatic brain injury: a multicenter cohort study. Neurocrit Care. 2013;18(1):154-60.

9. Puffer RC, Yue JK, Mesley M, et al. Recovery trajectories and long-term outcomes in traumatic brain injury: a secondary analysis of the phase 3 Citicoline Brain Injury Treatment Clinical Trial. World Neurosurg. 2019;125:e909-15.

10. Vedantam A, Robertson CS, Gopinath SP. Clinical characteristics and temporal profile of recovery in patients with favorable outcomes at 6 months after severe traumatic brain injury. J Neurosurg. 2018;129(1):234-40.

11. Kowalski RG, Hammond FM, Weintraub AH, et al. Recovery of consciousness and functional outcome in moderate and severe traumatic brain injury. JAMA Neurol. 2021;78(5):548-57.

12 Gregson BA, Mendelow AD, STICH Investigators. International variations in surgical practice for spontaneous intracerebral hemorrhage. Stroke. 2003;34(11):2593-7.

13. Fahlstrom A, Tobieson $L$, Redebrandt HN, et al. Differences in neurosurgical treatment of intracerebral haemorrhage: a nation-wide observational study of 578 consecutive patients. Acta Neurochir (Wien). 2019;161(5):955-65.

14 Merton RK. The self-fulfilling prophecy. Antioch Rev. 1948;8(2):193-210.

15. Weimer JM, Nowacki AS, Frontera JA. Withdrawal of life-sustaining therapy in patients with intracranial hemorrhage: Self-fulfilling prophecy or accurate prediction of outcome? Crit Care Med. 2016;44(6):1161-72.
16. Wilkinson D. The self-fulfilling prophecy in intensive care. Theor Med Bioeth. 2009;30(6):401-10.

17. Hwang DY, Chu SY, Dell CA, et al. Factors considered by clinicians when prognosticating intracerebral hemorrhage outcomes. Neurocrit Care. 2017;27(3):316-25

18. Larach DR, Larach $D B$, Larach MG. A life worth living: seven years after craniectomy. Neurocrit Care. 2009;11(1):106-11.

19. Creutzfeldt CJ, Holloway RG. Treatment decisions after severe stroke: uncertainty and biases. Stroke. 2012;43(12):3405-8.

20. Chapman AR, Litton E, Chamberlain J, et al. The effect of prognostic data presentation format on perceived risk among surrogate decision makers of critically ill patients: a randomized comparative trial. J Crit Care. 2015;30(2):231-5.

21. Boyd EA, Lo B, Evans LR, et al. "It's not just what the doctor tells me:" factors that influence surrogate decision-makers' perceptions of prognosis. Crit Care Med. 2010;38(5):1270-5.

22. Garg A, Soto AL, Knies AK, et al. Predictors of surrogate decision makers selecting life-sustaining therapy for severe acute brain injury patients: an analysis of US population survey data. Neurocrit Care. 2021. https://doi. org/10.1007/s12028-021-01200-9.

23. Hwang DY, Knies AK, Mampre D, et al. Concerns of surrogate decision makers for patients with acute brain injury: a US population survey. Neurology. 2020;94(19):e2054-68.

24. Weber U, Zhang Q, Ou D, et al. Predictors of family dissatisfaction with support during neurocritical care shared decision-making. Neurocrit Care 2021. https://doi.org/10.1007/s12028-021-01211-6.

25. White DB, Angus DC, Shields AM, et al. A randomized trial of a family-support intervention in intensive care units. N Engl J Med. 2018;378(25):2365-75.

26. Davidson JE, Aslakson RA, Long AC, et al. Guidelines for familycentered care in the neonatal, pediatric, and adult ICU. Crit Care Med. 2017:45(1):103-28.

27. Creutzfeldt CJ, Engelberg RA, Healey L, et al. Palliative care needs in the neuro-ICU. Crit Care Med. 2015;43(8):1677-84.

28. Han JJ, Raiten JM. Escaping the labyrinth - on finding a common path forward in the ICU. N Engl J Med. 2021;384(24):2269-71.

29. Wijdicks EF, Rabinstein AA. The family conference: end-of-life guidelines at work for comatose patients. Neurology. 2007;68(14):1092-4. 